\title{
Electrochemistry in an optical fiber microcavity - optical monitoring of electrochemical processes in picoliter volumes
}

Tomasz Gabler ${ }^{1, \dagger}$, Andrzej Krześniak ${ }^{2, \dagger}$, Monika Janik ${ }^{1,3}$, Anna Myśliwiec ${ }^{1}$, Marcin Koba ${ }^{1,4}$, Joanna Buczyńska ${ }^{2}$, Martin Jönsson-Niedziółka ${ }^{2, *}$, and Mateusz Śmietana, ${ }^{1, *}$

1. Warsaw University of Technology, Institute of Microelectronics and Optoelectronics, Koszykowa 75, 00-662 Warsaw, Poland

2. Polish Academy of Sciences, Institute of Physical Chemistry, Kasprzaka 44/52, 01-224

Warsaw, Poland

3. Gdańsk University of Technology, Department of Metrology and Optoelectronics, Gabriela Narutowicza 11/12, 80-233 Gdańsk, Poland

4. National Institute of Telecommunications, Szachowa 1, 04-894 Warsaw, Poland

*E-mail: M.Smietana@elka.pw.edu.pl, martinj@ichf.edu.pl

$\dagger$ These people contributed equally to the work.

Keywords: optical fiber sensor, electrochemistry, interferometers, laser micromashining, optical properties

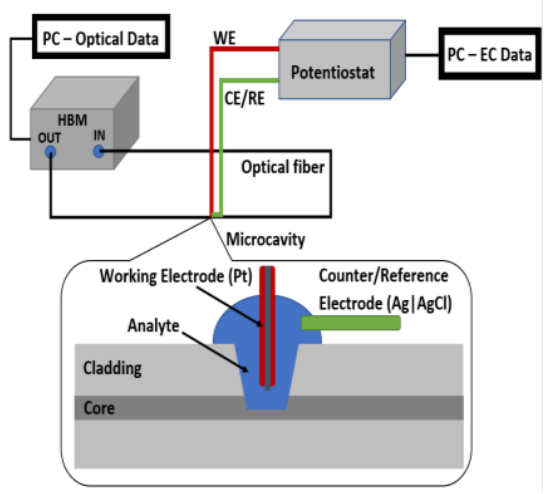

\begin{abstract}
In this work, we demonstrate a novel method for multi-domain analysis of properties of analytes in volumes as small as picoliter, combining electrochemistry and optical measurements. A microcavity in-line Mach-Zehnder interferometer ( $\mu \mathrm{IMZI}$ ) obtained in a standard single-mode optical fiber using femtosecond laser micromachining was able to accommodate a microelectrode and optically monitor electrochemical processes inside the fiber. The interferometer shows exceptional sensitivity to changes in optical properties of analytes in the microcavity. We show that the optical readout follows the electrochemical reactions. Here, the redox probe (ferrocenedimethanol) undergoing reactions of oxidation and reduction changes the optical properties of the analyte (refractive index and absorbance) that are monitored by the $\mu$ IMZI. Measurements have been supported by numerical analysis of both optical and electrochemical phenomena. On top of a capability of the approach to perform analysis in microscale, the difference between oxidized and reduced forms in the near-infrared can be clearly measured using the $\mu$ IMZI, which is hardly possible using other optical techniques. The proposed multi-domain concept is a promising approach for highly reliable and ultrasensitive chemo- and biosensing.
\end{abstract}




\section{Introduction}

When investigation of liquids is concerned, electrochemical (EC) and optical domains can be found as the most often explored [1]. EC sensors rely on the exchange of charges between an electrode and an analyte or mediator molecule. These sensors can work in many different modes, e.g., amperometric, voltametric, and potentiostatic [2]. EC approaches may offer exceptionally high sensitivity to alteration of electrode surface and its proximity, that may be caused by specific binding of certain biomolecules to biofunctionalized surface of the electrode [3]. Optical sensors in turn exploit the interaction of light with matter in two steps - first in the interaction with the analyte to create an analytic signal, and then with an optical transducer to transform the optical response to an electric signal. Optical systems offer unique advantages, such as immunity to electromagnetic interferences, degradation, or corrosion effects, which are often problematic for EC sensors. Moreover, optical interactions with an analyte may take place further from the sensor surface then in case of EC sensors, what make possible optical investigations of e.g., large biological targets. Due to numerous advantages of sensors operating in different domains, multi-domain sensing methods have been gaining in popularity, as they can simultaneously probe different properties of the targeted sample at the same time and spot, and thereby provide parallel information about multiple parameters that would not be available with the respective techniques alone [4-6]. On top of enhanced information about the analyte, the multi-domain methods may offer a capability for cross-verification of the readouts received in each of the domains alone This makes the results of the measurement highly reliable [7].

The number of solutions developed to date for multi-domain sensing is significantly smaller than that available for single-domain ones. One of these multi-domain sensing strategies, but highly explored, is the combination of EC and optical systems. Perhaps the most common of these methods is electrochemiluminescence, where the reaction between the analyte and a luminophore generates a species that can undergo high-energy electron-transfer to lightemitting excited states [8]. Other examples include combined EC and plasmonic sensing methods, such as surface plasmon resonance (SPR) or localized SPR [9], as well as methods linking EC with fluorescence [10] or colorimetry [11]. Arguably the most well-knows combination of optics with EC is spectroelectrochemistry (SEC). While sometimes used as a sensing strategy [12], the method is most frequently used for investigation of reaction mechanisms or kinetic studies. The future of this field was recently reviewed by Lozeman et al. [13], where they focused on the, in their opinion, most promising methods, such as infrared SEC and Raman-SEC. A novel example of combining optical and EC methods was explored by Śmietana et al. where they used lossy-mode resonance effect-based optical fiber probe as a working electrode in EC domain [7]. Sensing approaches based on an optical fiber on top of high sensitivity reached in optical domain offer sensing system miniaturization.

Highly limited availability of studied liquids in analytical chemistry and biology is a key driver for miniaturization of sensing devices and systems. However, it must be noted that behavior of the systems on a micro- or nanoscale often differs from those in bulk volumes, e.g., in EC observed as higher diffusion limited currents, lower capacitive effects and faster response times [14]. This has been explored in EC using micro- and nanoelectrodes (formerly known as ultramicroelectrodes [15]) since the 1980s [16,17]. Similarly, measurements in tiny volumes show different responses compared to standard EC in bulk solutions [18]. EC cells with picoliter volumes were studied by Clark et al., using cells etched in polystyrene [19]. Small volume measurements have been realized in numerous ways over the years. Femto- and attoliter cells were made by photolithographic processing on microchips [20]. EC processes in femtoliter droplets have been studied for years through the transient current as they collide with a carbon microelectrode [21,22]. Using recessed carbon electrodes in a nanopipette, a so-called nanosampler [23], Mirkin and co-workers could reach zeptoliter volumes and through generation-collection cycling study single molecule redox processes [24]. However, it has been 
noticed that in open EC cells some additional issues must be faced, i.e., evaporation of the electrolyte becomes crucial as surface to volume ratio increases. The problem can be ameliorated to a certain degree by covering the sample with a non-volatile organic solvent, such as heptane or mineral oil [25,26]. The sample solution can also be mixed with e.g., glycerin to slow down the evaporation [27]. Moreover, in miniaturized condition introduction of additional sensing technique to make multi-domain sensing possible and increase reliability of the measurements is highly challenging.

The development of microfabrication techniques such as laser ablation introduced new sensing approaches, which include microcavities induced in an optical fiber. The presence of the microcavity with the size of tens of micrometers is the defining feature of these platforms. One of the reported configurations is a microcavity in-line Mach-Zehnder interferometer ( $\mu$ IMZI) [28]. The interferometric structure is formed by a well-controlled fiber cladding, and partially core, femtosecond (fs) laser ablation. The microstructure splits light guided in the fiber core into two parts, where one continues to propagate inside the remaining part of the core, while the other propagates through the microcavity. The two beams interfere at the far sidewall of the cavity resulting in an interference pattern observed in the fiber transmission spectrum. Thanks to the ablated part of the fiber core, the analyzed media can directly interact with the light what justifies remarkably high refractive index (RI) sensitivity for this approach reaching over 20,000 RIU/nm [29]. Till now, the $\mu$ IMZI sensor has been characterized in terms of fabrication and its optimization utilizing, e.g., reactive ion etching [30], or fs laser postprocessing [29]. The sensitivity of the system has been investigated with the use of the thin layer deposition [31]. Although the $\mu$ IZMI has been reported for different applications, such as bacteria detection [32, 33], and real-time monitoring of isothermal DNA amplification [34], the subject of using the in-fiber sensing structures for multi-domain measurements has never been addressed or studied in the literature reported so far.

In this work, we demonstrate, for the first time the multi-domain, i.e., combined optical and EC measurements, in volumes as small as picoliter. The $\mu$ IMZI structure obtained in an optical fiber was able to accommodate microelectrode and EC processes inside the fiber. We show that the optical readout follows the EC reactions, what makes the sensing concept a unique approach for combined opto-EC analysis in micro-scale.

\section{Results and discussion}

In Figure 1 the $\mu$ IMZI used in this experiment is shown. It is important to emphasize that the whole surface of the microcavity is well-defined, and no damages were introduced to the fiber outside the cavity during the microfabrication. The microelectrode, also when inserted into the microcavity, is shown in Figure 2A. It can be seen that the shape of the microelectrode tip fits well the microcavity. After inserting the microelectrode into the microcavity, optical and electrochemical measurements were done simultaneously in a setup shown schematically in Figure 2B. 

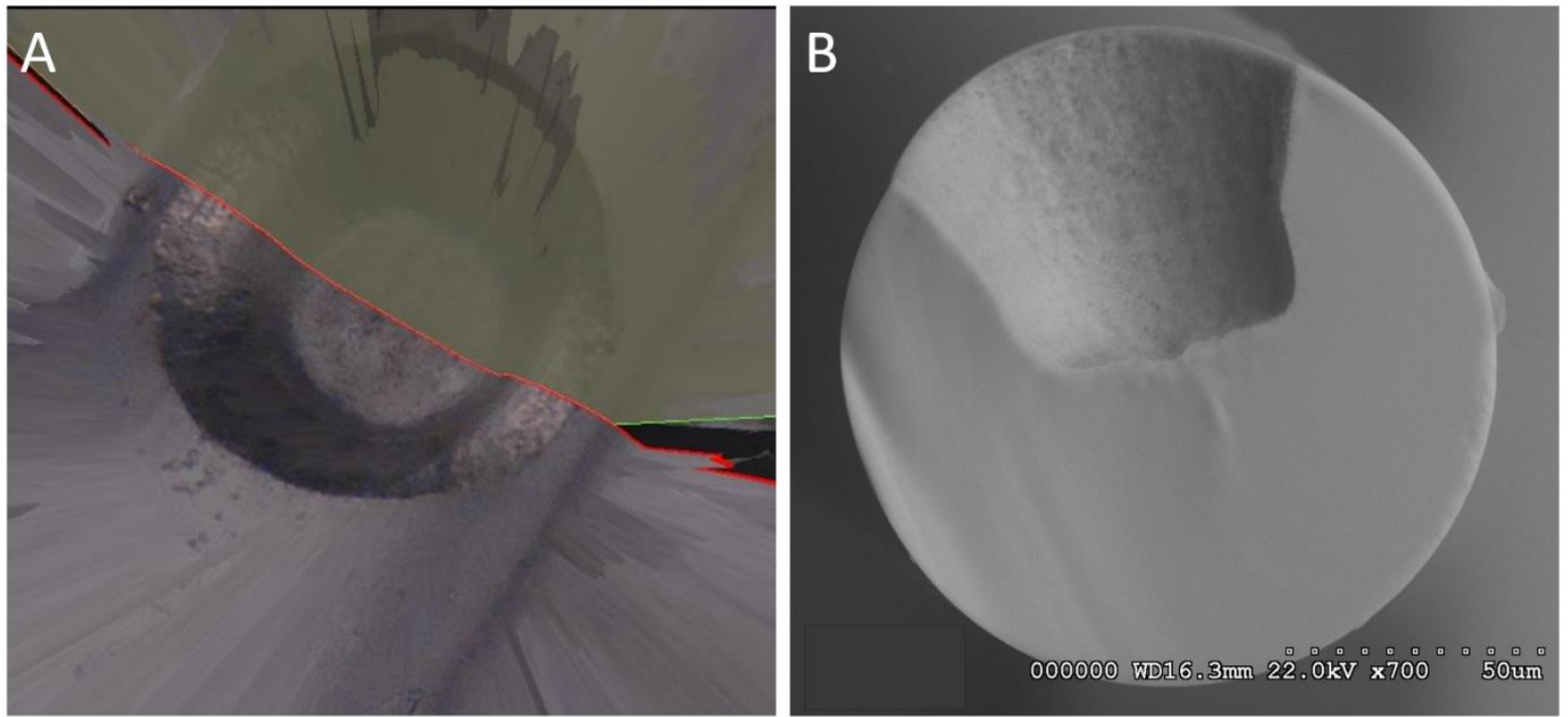

Figure 1. (A) The top-view of the in-fiber microcavity captured by confocal microscope. The red line indicates approximate place of the structure's cross-section depicted in (B) which shows an image captured by a scanning electron microscope.
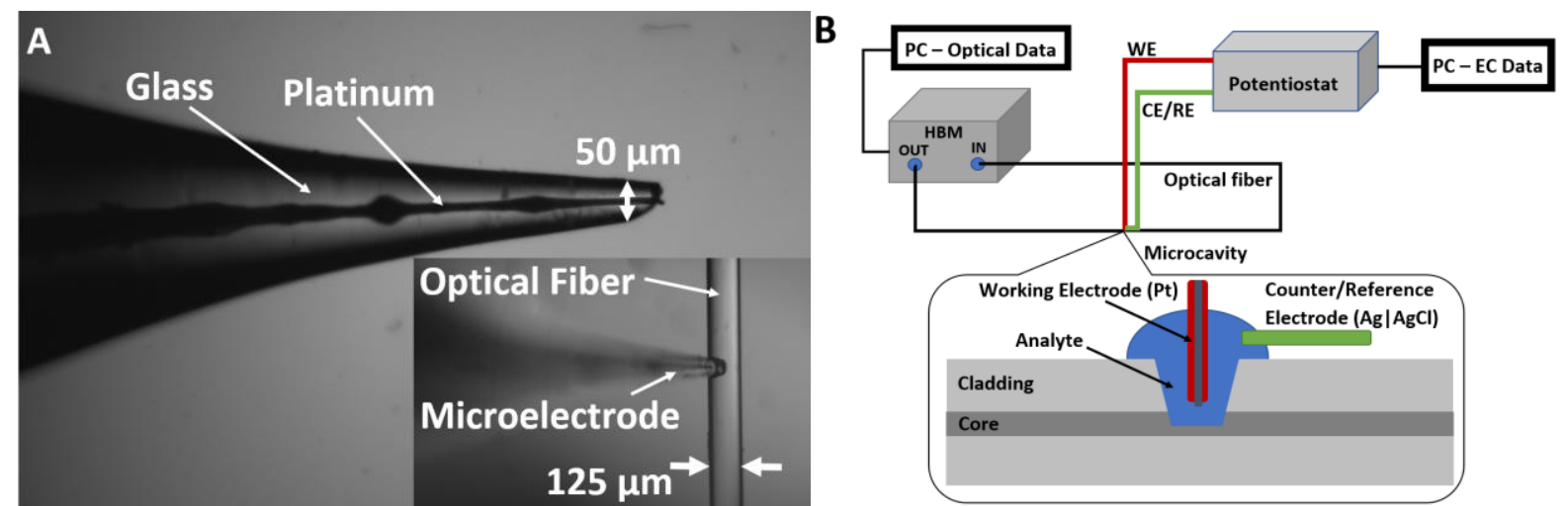

Figure 2. (A) Microscopic image of the microelectrode and (B) schematic showing experimental setup. Inset in (A) shows microelectrode inserted in the microcavity.

As the RI sensitivity of the fabricated $\mu$ IMZI is crucial for optical monitoring of EC, it has been identified right after the microfabrication. Figure 3A presents the transmission spectra of the chosen $\mu \mathrm{IMZI}$ for which the RI filling the cavity varied from 1.33292 to 1.34018 RIU. Both, wavelength corresponding to the minima as well as transmitted power change with RI. The value of transmission increases, and the minima shift towards shorter wavelengths as the RI increases. The $\mu$ IMZI described in this work, for the RI filling the microcavity slightly higher than that of water, displays the RI sensitivities ranging from approximately $10,000 \mathrm{~nm} / \mathrm{RIU}$ up to almost $14,000 \mathrm{~nm} / \mathrm{RIU}$ for the minimum at shorter and longer wavelengths, respectively. Moreover, a small or negligible deviation of measured points from approximation lines can be noticed. This reveals high linearity of the sensitivity (Figure 3A, inset). Figure 3B, where the part of the spectrum corresponding to minimum at longer wavelengths is plotted, shows that the presence of the microelectrode in the microcavity has also an impact on the transmission pattern. The deeper the microelectrode is inserted in the microcavity, the more the pattern corresponds to that when RI increases, i.e., the minimum shifts towards shorter wavelengths and the transmission increases. 


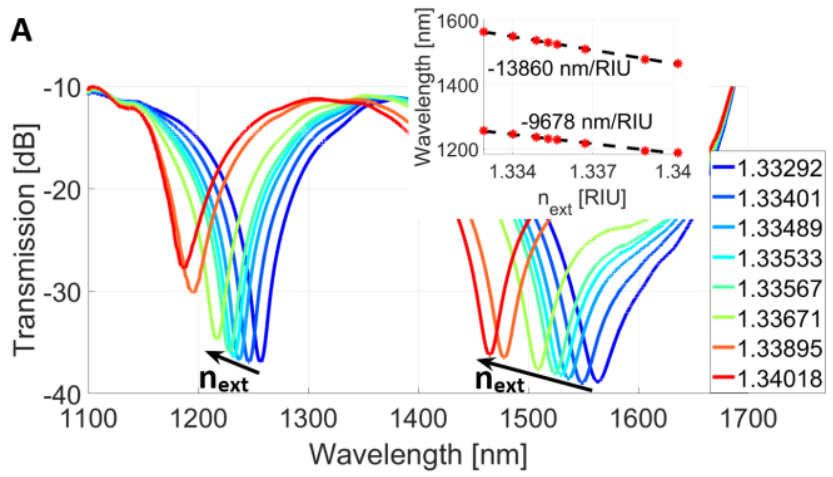

B

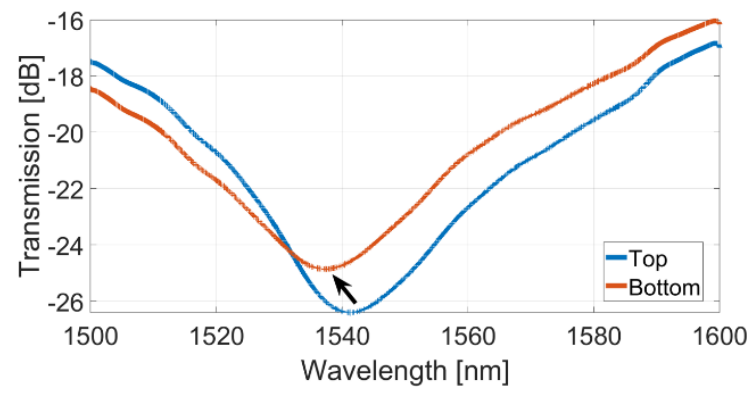

Figure 3. (A) Response of the spectral response of the $\mu$ IMZI on external RI (next) and (B) impact of the microelectrode insertion into the cavity for two positions of the microelectrode tip, namely at the top of the microcavity entrance (blue line) and at its bottom (red line). The ranges of transmission and wavelength differ in (A) and (B) due to distinct measurement setups used in the two cases.

In Figure 4 results of numerical calculations of the electromagnetic field distribution at the $\mathrm{X}-\mathrm{Y}$ plane in the middle of the microcavity are shown. They depict a beam-splitting effect at the microcavity wall. The light is guided in the residual core (below the dashed line), as well as in the microcavity (close to its bottom). However, the intensity of light in the latter is but slightly lower than that in the former. The results indicate that the light guiding in the microcavity can be meaningfully disturbed when the microelectrode is up to approx. $10 \mu \mathrm{m}$ above the microcavity bottom.

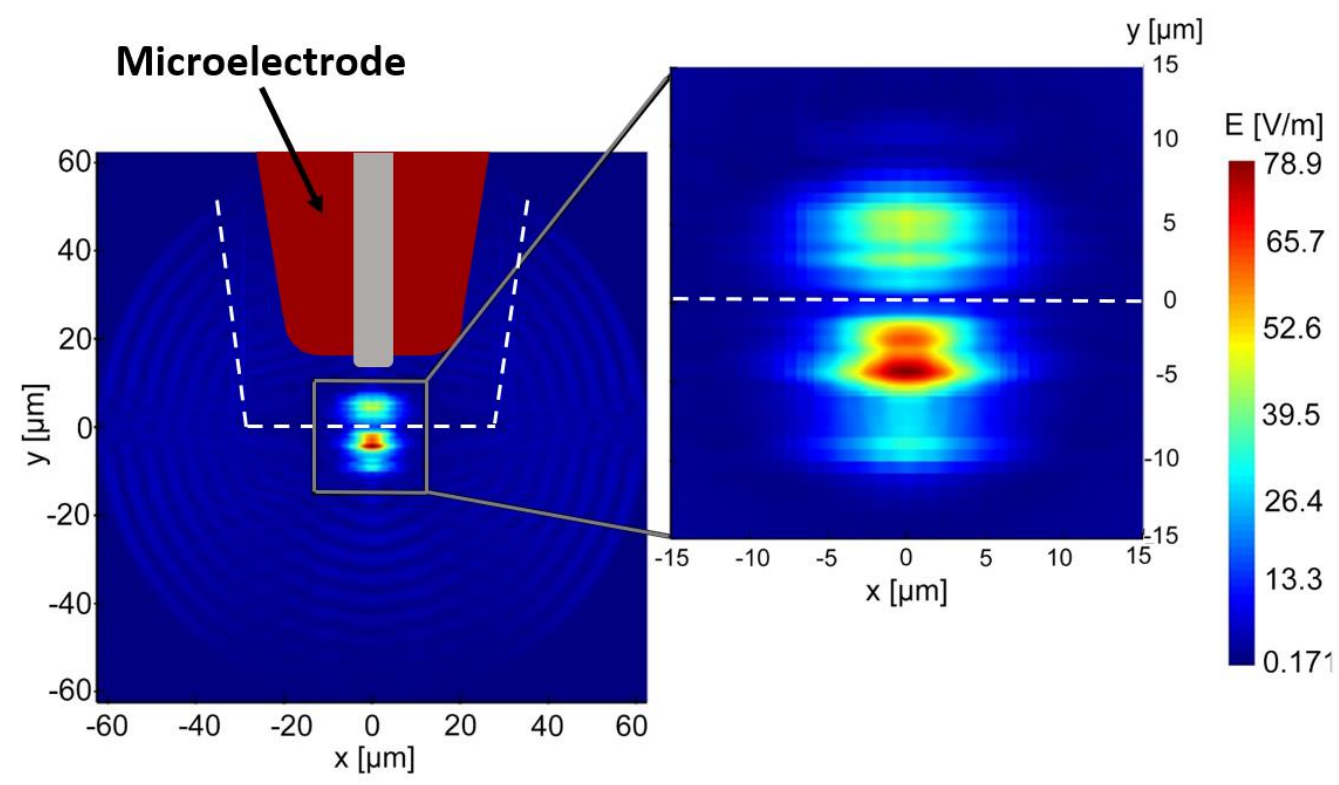

Figure 4. X-Y cross-section of an optical fiber with electromagnetic field distribution plotted in the middle of the microcavity. The calculations were run for the RI filling the microcavity set to $1.33 \mathrm{RIU}$ and the wavelength to $1554 \mathrm{~nm}$. Microelectrode inserted in the microcavity is shown schematically for reference purposes.

Next, a series of multistep chronoamperometric (CA) experiments in parallel to optical measurements was performed for different positions of the microelectrode. First series of the experiments done for microelectrode tip at the bottom of the microcavity was followed by the second series, when the tip was in the central part of the microcavity. For each location, the potential was switched between $0.0 \mathrm{~V}$ and $0.4 \mathrm{~V}$. These values were kept constant for $100 \mathrm{~s}$ and the switching was repeated ten times. The effect of CA performed in the microcavity on both optical and EC responses is shown in Figure 5. For the EC response (Figure 5, inset) an increase in oxidation current (for potential $0.4 \mathrm{~V}$ ) after 2000 s reached around $10 \%$, which corresponds 
to a slight evaporation of the droplet and increase in concentration of the redox probe contributing to the measured current. Since the experiment for the electrode at the bottom and in the central part of the microcavity followed one another, the increase in current induced by the electrolyte evaporation for the electrode in the central part progressed. On top of changes in current induced by switching of the potential, there is also an impact of the potential on the optical response. In Figure 5 changes in transmission at its minimum were shown due to high sensitivity of the optical spectrum analyzer in power domain. Shift of the minimum in wavelength domain took place too, but due to relatively broad minimum it was difficult to be identified. Figure S6 in Supplementary Information shows the shift of the slope measured at fixed transmission and the shift in wavelength corresponds well with the one for transmission shown in Figure 5. In general, elevated potential increases transmission of the resonance minimum and its shift towards shorter wavelength, the effect similar as the one when RI in the microcavity increased. Moreover, the progressing shift of transmission and wavelength during entire experiment is seen. The effect can correspond to two factors, namely drift in time of the light source in the optical interrogation system and indicated above, evaporation of the electrolyte. To verify an impact of the first factor long-term measurements of transmission via reference SMF patchcord were performed at $1550 \mathrm{~nm}$. The results shown in Figure S6 in Supplementary Information indicate that the drift is the most significant during initial 30 minutes of the system operation. With time the drift is still measurable, but way less meaningful. Thus, all the measurements were performed with the optical interrogation system initially heated-up for at least 30 minutes.

Since the drift is relatively insignificant, in optical domain the effect of the electrolyte evaporation can be noticed, similarly as in the case of EC response. An increase in transmission corresponds to increase in RI of the analyzed solution. What is interesting, the dynamics of the evaporation at the microcavity bottom and in its central part seem different. At the bottom, the electrolyte densification progresses during the experiment, but when the electrode is in the central part at the beginning a delayed process, is noticed. The difference in power levels at the bottom and in the central part of the cavity shows the impact of rather large electrode. While at the bottom, it acts constructively by disrupting the scattering in the liquid and improves, although not significantly, coupling of the light back to the fiber.

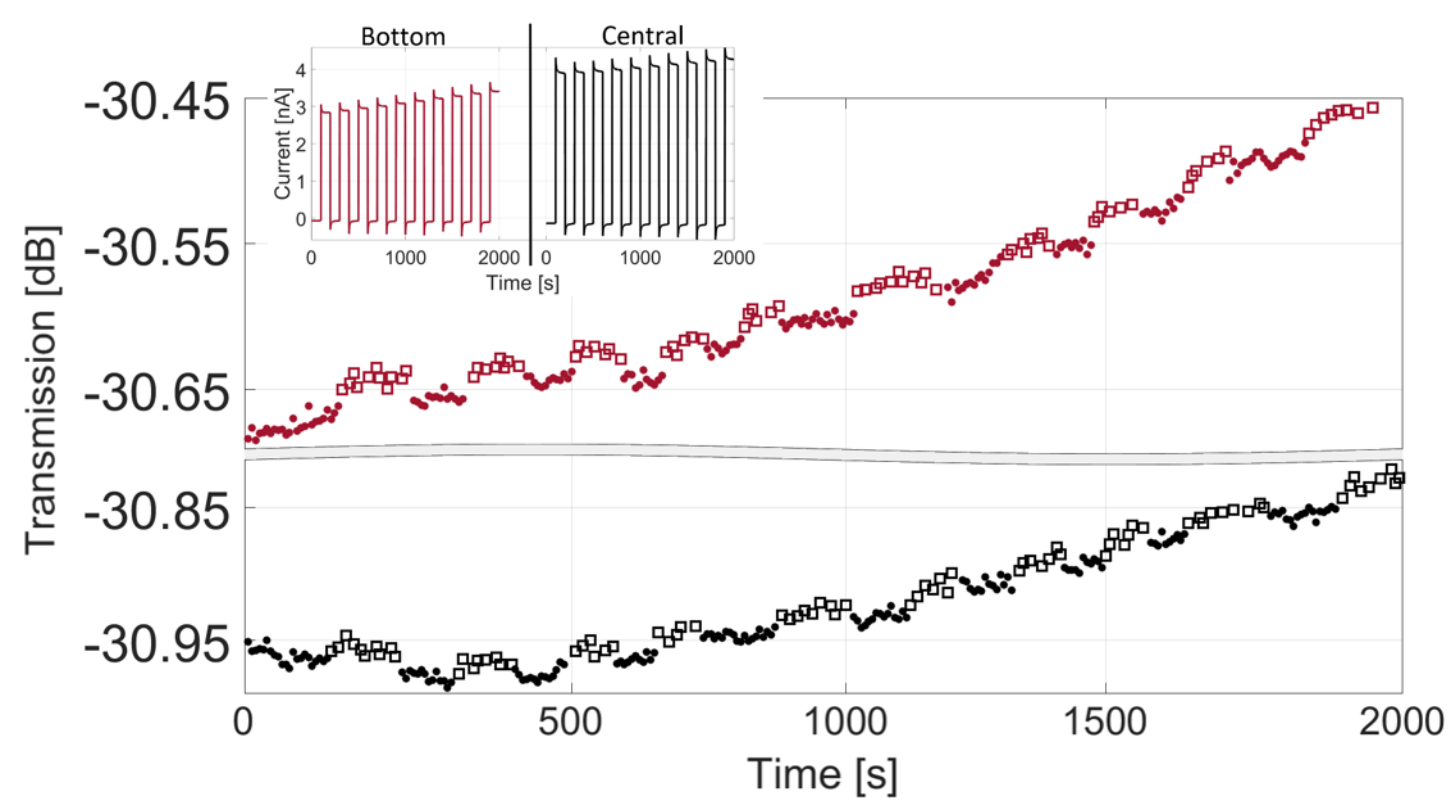

Figure 5. Optical and EC (inset) response during CA measurements for the microelectrode tip located at the bottom (red) and central part (black) of the microcavity. Open and close shapes correspond to $0.4 \mathrm{~V}$ and $0.0 \mathrm{~V}$ potential, respectively. 
Following CA, also cyclic voltammetry (CV) measurements have been performed (Figure 6). For $\mathrm{CV}$ we used a scan rate of $20 \mathrm{mV} / \mathrm{s}$ and potential ranging from $-0.3 \mathrm{~V}$ to $0.5 \mathrm{~V}$. The main difference between the results for the position of the microelectrode at the bottom of the microcavity and $30 \mu \mathrm{m}$ from the bottom is the steady-state current, which is around $8 \%$ lower at the bottom. This is an effect of geometrical inhibition of the diffusion of the electroactive ions between the electrodes when the size of working electrode is comparable to the size of microcavity. The effect of evaporation can be neglected in this case, as the time scale of the CV experiments was much shorter than that of the CA.

A

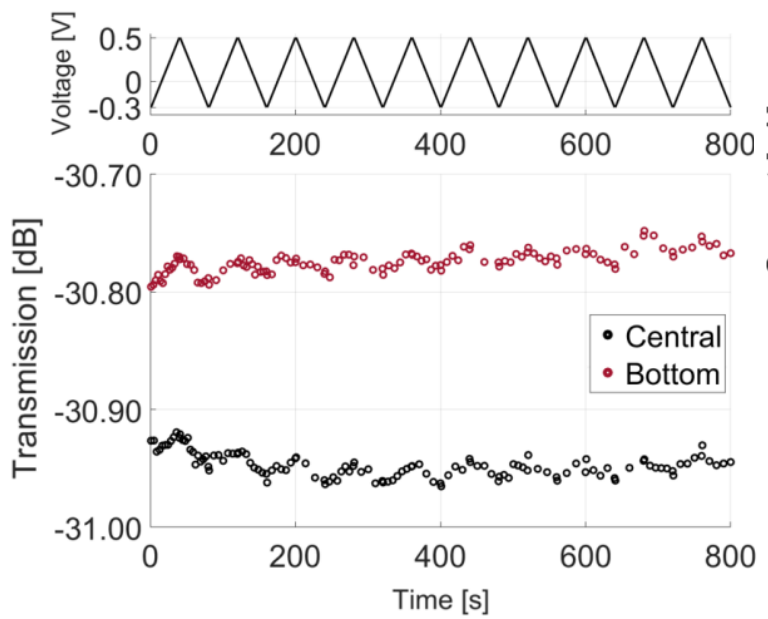

B

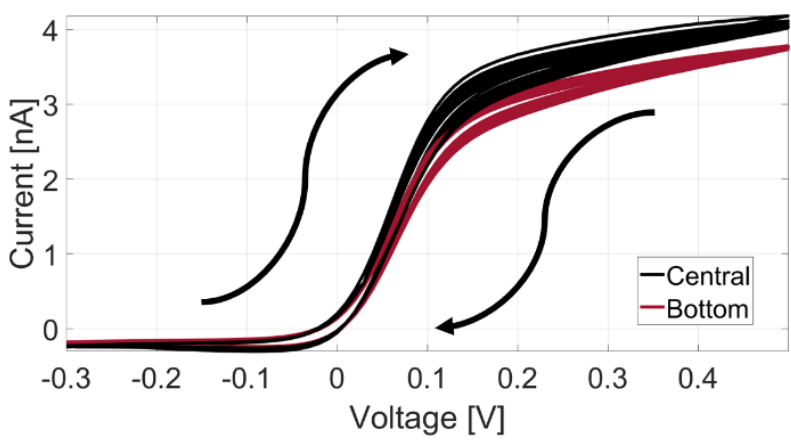

Figure 6. (A) Optical and (B) EC response during CV measurements for the microelectrode tip located at the bottom (red) and central part (black) of the microcavity.

Figure 7 shows the results of the simulations of the CA measurements. The current response for the electrode in the middle of the microcavity (electrode $30 \mu \mathrm{m}$ above the bottom) is very close to the measured curve. However, the current measured at the bottom of the microcavity is significantly smaller in the simulations than in the experimental data. The reason is likely that the electrode was inserted into the cavity at a slight angle or not centrally, or a combination of the two, both of which lead to an increased diffusion on one side of the electrode. From Figure 7B we see that despite a lower current when the electrode is at the bottom, the concentration changes slightly more in the sampling volume. We can also see that the response is very fast, which agrees well with the experimental results in Figure 5. Simulations for CVs can be found in Section 1 in the Supplementary Information.

A

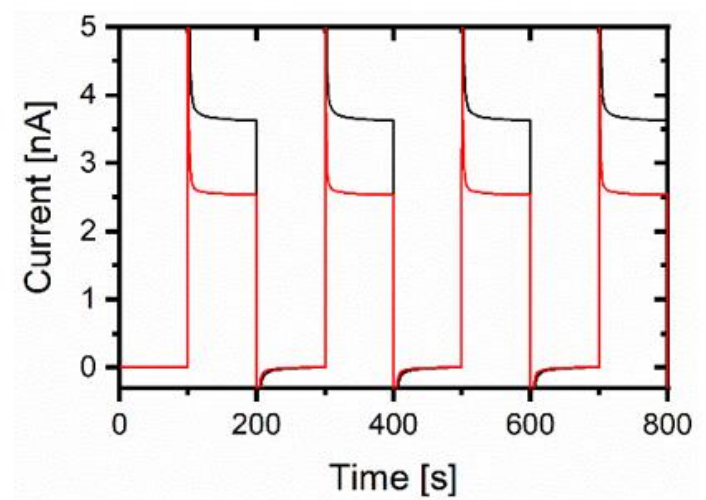

B

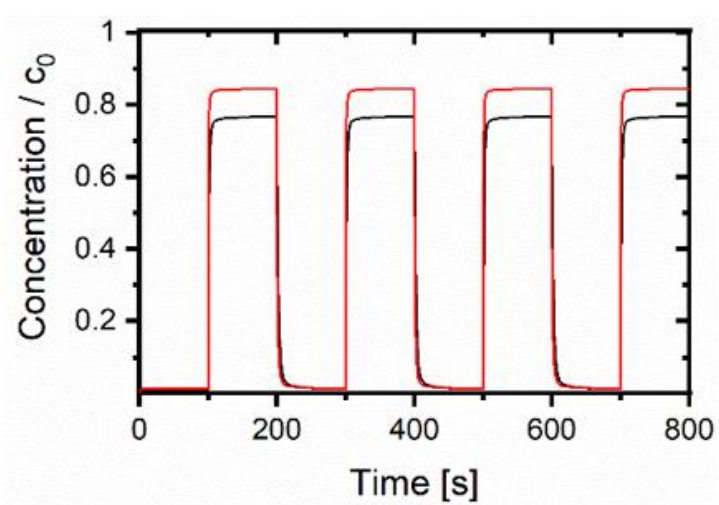

Figure 7. (A) Simulated CAs and (B) the corresponding changes in concentration of the oxidized form of the redox probe. Black lines are for the electrode in the central of the cavity $(\mathrm{h}=30 \mu \mathrm{m})$ and red with the electrode close to the bottom $(\mathrm{h}=7 \mu \mathrm{m})$. 
Comparison of the results from the simulations of CV (Figure S2 in Supplementary Information) and CA (Figure 7) shows that the change in concentration between the oxidized and reduced forms of the redox probe in the sampling volume close to the bottom of the cavity are similar in the two sets of experiments and corresponds to about $80 \%$ of the redox probe being in its oxidized form. This is also reflected in the response in the optical signal, which is of similar magnitude in both cases. The simulations also show that the effect on concentration is higher when the electrode is further inside the cavity, despite the oxidation current, and thus total amount of oxidized form of the redox probe, being smaller. This is because the oxidation happens closer to the sampling volume. The difference is quite small, but the effect can be seen in the optical data, where the amplitude of transmitted power change is slightly bigger in the case where the electrode is deeper in the cavity (Figure 5).

To identify possible origins of the potential-induced modulation of optical response observed in Figure 5A and 6A a short theoretical background of IMZI operation is outlined below. In general, for this kind of interferometers the intensity $(I)$, which is proportional to the transmitted power, is expressed through:

$$
I=I_{\text {core }}+I_{\text {cavity }}+2 \sqrt{I_{\text {core }} I_{\text {cavity }}} \cos \left(\frac{2 \pi d\left(n_{\text {eff }}^{\text {core }}-n_{\text {eff }}^{\text {cavity }}\right)}{\lambda}+\varphi_{0}\right)
$$

where $I_{\text {core }}$ and $I_{\text {cavity }}$ are the intensities of waves propagating in two interferometric paths, i.e., along the core and in the cavity, $d$ is a length of the cavity, $n_{\text {eff }}^{\text {core }}-n_{\text {eff }}^{\text {cavity }}$ is a difference between effective RI for waves in core and the microcavity, $\lambda$ is wavelength and $\varphi_{0}$ is an initial phase [35]. After straightforward transformations [35], the interference fringe pattern has minima $\lambda_{m}$ for every odd multiple of $\pi$ :

$$
\lambda_{m}=\frac{2 \pi d\left(n_{\text {eff }}^{\text {core }}-n_{\text {eff }}^{\text {cavity }}\right)}{(2 m+1) \pi-\varphi_{0}}
$$

When RI in the microcavity increases it has an impact on the $n_{\text {eff }}^{\text {cavity }}$ and induces shift of the interference pattern towards shorter wavelengths. To verify possible changes in RI a reference experiment has been performed using the automatic refractometer. It works in visible spectral range, while infrared properties are concerned in the microcavity. However, according to [40] the RI of the solution is lower in infrared than in visible, and the changes keep the same trend for the two wavelength ranges. The experiment has proven that oxidation of the investigated solution, as well as other solutions with redox probes (see Section 4.1 of Supplementary Information for details), increases their RI. However, even taking into consideration high RI sensitivity of $\mu \mathrm{IMZI}$ the shift in wavelength of the minimum would not exceed $1 \mathrm{~nm}$.

In reported in this work CV and CA experiments, changes in the transmitted power at its minimum are better visible than those of wavelength and they correlate to the changes of the applied potential too. It must be noted that in general when absorbance of the investigated solution increases it decreases all terms involving $I_{\text {cavity }}$ and therefore decreases the transmitted optical power. Thus, reference measurements of absorbance of $\mathrm{KCl}$ solution with and without the redox probe have been performed (see Section 4.2 in Supplementary Information for details) and showed no significant difference between these solutions. However, absorbance of any water-based solution at ca. $1400-1600 \mathrm{~nm}$ is very high, and it makes standard absorbance measurements unreliable [36,37]. Taking into consideration that the interaction length of light and the solution in the microcavity does not exceed $60 \mu \mathrm{m}$, the application of the $\mu$ IMZI may be considered as a unique technique for identification of minute changes in both RI and absorbance.

As concluded above, the change in oxidation state for redox probe is the most likely explanation for the variation of the optical response. However, the very reason that 1,1'ferrocenedimethanol $(\mathrm{FcDM})$ is such a popular redox probe in EC makes it particularly 
challenging for interferometric measurements. The change in structure between the FcDM and the oxidized $\mathrm{FCDM}^{+}$is quite small [38]. Therefore, the difference in optical properties in the NIR region between the two forms is very small too, but still measurable using $\mu$ IMZI. It is therefore a testament to the sensitivity of the present method that the variation in optical response can be measured in the minute volume of the microcavity.

\section{Conclusion}

Here we have introduced a new multi-domain sensing method combining optical and electrochemical techniques. The method is using electrochemical oxidation of an analyte inside a microcavity induced in an optical fiber using femtosecond laser ablation. The cavity constitutes one path of a microcavity in-line Mach-Zehnder interferometer ( $\mu$ IMZI). These have been shown to be extremely sensitive to minute changes in refractive index of the liquid in the cavity [30]. We demonstrate this method using ferrocenedimethanol as a redox probe and monitor the changes in optical transmission pattern as the redox probe undergoes cycles of oxidation and reduction, using both cyclic voltammetry and chronoamperometry. The $\mu$ IMZI is based on commercial single-mode communications fiber so the operation wavelength is at ca $1550 \mathrm{~nm}$. At this wavelength, the complex refractive index is dominated by the absorption by water molecules, but our experiments show that even with a redox system where the optical spectrum in the near infrared cannot be distinguished from the background electrolyte, as is the case of ferrocenedimethanol, the difference between oxidized and reduced form can still clearly be measured using the $\mu$ IMZI. Considering these findings, when highly limited volume of an analyte is available, the electrochemically enhanced $\mu$ IMZI can be considered as a unique and highly sensitive approach for multi-domain chemo- and biosensing.

\section{Experimental Section}

\subsection{Fabrication of the microcavities}

The microcavities (54 $\mu \mathrm{m}$ diameter, $62.5 \mu \mathrm{m}$ height) were fabricated in a side surface of standard Corning SMF28 fibers. The micromachining process was performed using an NKT Origami 10XP laser operating at wavelength of $1030 \mathrm{~nm}$. The fiber was irradiated by ca. 400 fs pulses. Fused silica glass has very low absorption at $1030 \mathrm{~nm}$, and therefore linear absorption of the laser radiation does not occur when the glass is irradiated by the laser beam [39]. The system worked with a repetition rate of $15 \mathrm{kHz}$. The laser beam was focused on the sample with a suitably designed micromachining setup based on the Newport mFab system. It was equipped with a $20 \mathrm{x}$ lens $(\mathrm{NA}=0.30)$. The laser pulse energy was equal to $6 \mathrm{~nJ}$. Fiber transmission was monitored during the process with an NKT Photonics SuperK COMPACT supercontinuum white light source and a Yokogawa AQ6370D optical spectrum analyzer working with $0.5 \mathrm{~nm}$ resolution. The fabrication process was controlled with an in-house developed software.

\subsection{Fabrication of the microelectrodes}

For fabrication of microelectrodes borosilicate glass capillaries with $2.0 \mathrm{~mm}$ outer and $0.4 \mathrm{~mm}$ inner diameter, as well as platinum wire $(99,95 \%$ metals basis, $50 \mu \mathrm{m}$ diameter, purchased from Alfa Aesar), were used. We used a micropipette puller PC-10 from Narishige for the preparation of the microelectrodes. First, the capillaries were cut to $10 \mathrm{~cm}$ length using a diamond knife, rinsed with acetone and water, and then dried with an argon stream. A $1.5 \mathrm{~cm}$ long Pt wire was put into the middle part of a capillary. Performed next procedure of microelectrodes fabrication involved three steps. In the first one a capillary with a Pt wire was thinned in the central part by $1 \mathrm{~mm}$ pulling at a temperature reaching $620^{\circ} \mathrm{C}$ followed by another $1 \mathrm{~mm}$ at $610^{\circ} \mathrm{C}$ (a dependence between temperature and "heat" parameter set at the puller can be found in [40]). 
A full available weight load was used. In the second step a Pt wire was sealed in the middle of a capillary under vacuum, i.e., both ends of the capillary were connected to the vacuum pump and kept for 90 seconds in the puller at $620^{\circ} \mathrm{C}$. A heating step was repeated for the position of the capillary ca. $2 \mathrm{~mm}$ above and ca. $2 \mathrm{~mm}$ below the center. At this stage the position of the capillary was blocked to prevent and pulling. The last step (made without vacuum) was a final pulling at $550^{\circ} \mathrm{C}$ during which a capillary was pulled in the middle until braking into two pieces. This way two electrodes were made and later carefully inspected using an optical microscope and cut to the proper diameter using microscissors. Finally, a copper wire was inserted into microelectrode to establish an electric contact between the platinum microwire and a potentiostat at the stage of EC measurements.

\subsection{Electrochemistry}

The EC response of the sensor has been studied using a portable PalmSens4 potentiostat connected to a computer equipped with PSTrace 5.6 software. Platinum microelectrode served as a working electrode and $\mathrm{Ag} / \mathrm{AgCl}$ wire was used as a pseudoreference electrode. All the experiments have been done in a standard redox probe i.e., $1 \mathrm{mM} \mathrm{1,1'-ferrocenedimethanol}$ $(\mathrm{FcDM})$ in $0.1 \mathrm{M} \mathrm{KCl}$. For the preparation of solutions, we used: $\mathrm{KCl}$ powder from $\mathrm{POCh}$ (pure p.a.), 98\% FcDM from ACROS Organics and deionized water from Millipore system. A microelectrode was inserted gently into microcavity using computer-driven micromanipulator and Nikon NARISHIGE ITS-FN1 optical microscope. The experimental setup, i.e., microcavity with the microelectrode, was installed inside a Faraday cage to cut off any electric noises. Conditions inside the cage (temperature and relative humidity) were controlled using a Laserliner 082.027A thermohigrometer with a data logger.

\subsection{Optical measurements}

The spectral responses of the $\mu$ IMZI during its initial testing were measured in wide wavelength range, i.e., from 1100 to $1700 \mathrm{~nm}$, using Yokogawa AQ6370B optical spectrum analyzer and Leukos SM30 supercontinuum white light laser source. The RI sensitivity was measured by immersing the sensors in glycerin/water solutions with the RI in the range of $\mathrm{n}_{\mathrm{D}}=1.33300-1.40100$ RIU. The $\mathrm{n}_{\mathrm{D}}$ of the solutions was determined using Rudolph J57 automatic refractometer. The transmission measurements were conducted at constant mechanical tension and temperature $\left(25^{\circ} \mathrm{C}\right)$. Later, during the $\mathrm{EC}$ measurements, the spectral response of the structures was investigated in the limited wavelength range, i.e. from 1500 to $1600 \mathrm{~nm}$, using a portable HBM FS22 spectrum analyzer with build in light source. The analyzer was supported by an in-house developed software.

\subsection{Numerical analysis}

Both electromagnetic and EC numerical analysis were performed in this work. The electromagnetic field distribution in the microcavity was calculated using the finite-difference time domain (FDTD) method implemented in the Ansys/Lumerical Device Suit software. The optical fiber was modeled using built-in cylindrical fiber structure with standard SMF dimensions. The RIs of core and cladding were based on data reported in [41]. The microcavity was modeled as a cylinder reaching halfway into the fiber core and filled with RI of 1.33 RIU. A mode light source covering the spectral range of 1100-1700 nm was placed inside the fiber core, $450 \mu \mathrm{m}$ before the microcavity. Varying mesh was used for calculation purposes, i.e., coarse of $0.77-0.77-0.2 \mu \mathrm{m}$ (along X-Y-Z axes) in the general simulation area and fine of 0.1 $0.1-0.2 \mu \mathrm{m}$ in the core. The diversification was introduced to achieve greater precision for intense electromagnetic fields. 
Numerical analysis of the EC response was performed using the Comsol 5.6 software with the Electrochemistry module (see Section 1 in Supplementary Information for details). The electrode reaction was modeled using the Butler-Volmer equation with the exchange current density following the mass action law [42]. The cavity and electrode geometries were described in $2 \mathrm{D}$ cylindrical rotation symmetric coordinates and the change in concentration of the oxidized form of the redox probe during the experiment was monitored as the average in a discshaped region close to the bottom of the cavity. The constraints of the rotational symmetry means that the shapes of the cavity or the sampling region do not completely match the real sample as shown in Figure 1. A control was done in a full 3D geometry with a sensing volume matching the one in Figure 5 along the axis of the optical fiber. This showed a very similar current to the 2D simulation and only slightly higher change of concentration, which is why the 2D geometry was used for the rest of the calculations.

\section{Acknowledgements}

This work was supported in Poland by the National Science Centre (NCN) under grants No. 2018/29/B/ST7/02552.

\section{References}

[1] J. Vetelino, A. Reghu, Introduction to Sensors, CRC Press, Boca Raton 2017.

[2] E. Bakker, Anal. Chem. 2004, 76, 328.

[3] Y. Wu, Tilley, R. D., J. J. Gooding, J. Am. Chem. Soc. 2019, 141(3), 1162.

[4] E. Eltzov, S. Cosnier, R.S. Marks, Expert Rev. Mol. Diagn. 2011, 11 (5), 533.

[5] C. M. Hill, D. A. Clayton, S. Pan, Phys. Chem. 2013, 15, 20797.

[6] J. Juan-Colas, A. Parkin, K. E. Dunn, M. G. Scullion, T. F. Krauss, S. D. Johnson, Nat. Commun. 2016, 7, 12769.

[7] M. Śmietana, M. Koba, P. Sezemsky, K. Szot-Karpińska, D. Burnat, V. Stranak, J. Niedziółka-Jönsson, R. Bogdanowicz, Biosens. Bioelectron. 2020, 154, 112050

[8] C. Ma, Y. Cao, X. Gou, J.-J. Zhu, Anal. Chem. 2020, 92, 431.

[9] A. B. Dahlin, B. Dielacher, P. Rajendran, K. Sugihara, T. Sannomiya, M. Zenobi-Wong, J. Vörös, Anal. Bioanal. Chem. 2012, 402, 1773.

[10] H. Yang, Z. Zhou, K. Huang, M. Yu, F. Li, T. Yi, C. Huang, Org. Lett. 2007, 9, 4729.

[11] A. Zloczewska, A. Celebanska, K. Szot, D. Tomaszewska, M. Opallo, M. JönssonNiedziolka, Biosens. Bioelectron. 2014, 54, 455.

[12] N. González-Diéguez, A. Colina, J. López-Palacios, A. Heras, Anal. Chem. 2012, 84, 9146.

[13] J. J. A. Lozeman, P. Führer, W. Olthuis, M. Odijk, Analyst. 2020, 145, 2482.

[14] R. Kashyap, M. Gratzl, Anal. Chem. 1998, 70, 1468.

[15] J. M. Pingarrón, J. Labuda, J. Barek, C. M. A. Brett, M. F. Camões, M. Fojta, D. B. Hibbert, Pure Appl. Chem. 2020, 92, 641.

[16] R. M. Wightman, Anal. Chem. 1981, 53, 1125A.

[17] A. Russell, K. Repka, T. Dibble, J. Ghoroghchian, J. J. Smith, M. Fleischmann, S. Pons, Anal. Chem. 1986, 58, 2961.

[18] R. A. Clark, A. G. Ewing, Anal. Chem. 1998, 70, 1119.

[19] R. A. Clark, P. B. Hietpas, A. G. Ewing, Anal. Chem. 1997, 69, 259.

[20] T. Li, L. Su, W. Hu, H. Dong, Y. Li, L. Mao, Anal. Chem. 2010, 82, 1521.

[21] R. Kazemi, N. E. Tarolla, J. E. Dick, Anal. Chem. 2020, 92, 16260.

[22] H. Deng, J. E. Dick, S. Kummer, U. Kragl, S. H. Strauss, A. J. Bard, Anal. Chem. 2016, 88, 7754. 
[23] Y. Yu, J.-M. Noël, M. V. Mirkin, Y. Gao, O. Mashtalir, G. Friedman, Y. Gogotsi, Anal. Chem. 2014, 86, 3365.

[24] P. Sun, M. V. Mirkin, J. Am. Chem. Soc. 2008, 130, 8241.

[25] K. P. Troyer, R. M. Wightman, Anal. Chem. 2002, 74, 5370.

[26] K. Yum, H. N. Cho, J. Hu, M.-F. Yu, ACS Nano. 2007, 1, 440.

[27] J. C. Ball, D. L. Scott, J. K. Lumpp, S. Daunert, J. Wang, L. G. Bachas, Anal. Chem. 2000, 72, 497.

[28] Y. Zhao, H. Zhao, R. Lv, J. Zhao, Opt. Lasers Eng. 2019, 117, 7.

[29] M. Janik, T. Eftimov, M. Koba, M. Smietana, W. J. Bock, J. Light. Technol. 2019, 37, 4501.

[30] M. Janik, M. Koba, A. Celebańska, W. J. Bock, M. Śmietana, Opt. Laser Technol. 2018, 103, 260.

[31] M. Śmietana, M. Janik, M. Koba, W. J. Bock, Opt. Express 2017, 25, 26118.

[32] M. Janik, M. Koba, A. Celebańska, W. J. Bock, M. Śmietana, Sci. Rep. 2018, 8.

[33] M. Janik, E. Brzozowska, P. Czyszczoń, A. Celebańska, M. Koba, A. Gamian, W. J. Bock, M. Śmietana, Sensors Actuators, B Chem. 2021, 330.

[34] M. Janik, S. V. Hamidi, M. Koba, J. Perreault, R. Walsh, W. J. Bock, M. Śmietana, Lab Chip 2021, 21, 397.

[35] E. Udd, ed., Fiber Optic Sensors: an Introduction for Engineers and Scientists, Wiley 2011.

[36] V. T. Hoang, D. Dobrakowski, G. Stępniewski, R. Kasztelanic, D. Pysz, K. D. Xuan, M. Klimczak, M. Śmietana, R. Buczyński, Opt. Mater. Express 2020, 28 (22/26) 32483.

[37] S. Kedenburg, M. Vieweg, T. Gissibl, H. Giessen, Opt. Mater. Express 2012, 2(11), 1588

[38] M. M. Flores-Leonar, R. Moreno-Esparza, V. M. Ugalde-Saldívar, C. Amador-Bedolla, Comput. Theor. Chem. 2017, 1099, 167-173

[39] J. Qiu, K. Miura, K. Hirao, J. Non-Cryst. Solids 2008, 354, 1100.

[40] J. Jedraszko, M. Michalak, M. Niedziółka-Jönsson, W. Nogala, J. Electroanal. Chem. 2018, 815, 231.

[41] P. Biswas, N. Basumallick, S. Bandyopadhyay, K. Dasgupta, A. Ghosh, S. Bandyopadhyay, IEEE Sens. J. 2015, 15, 1240.

[42] E. J. F. Dickinson, A. J. Wain, J. Electroanal. Chem. 2020, 872, 114145. 